\title{
Use of surrogate modelling for multiobjective optimisation of urban wastewater systems
}

\author{
G. Fu, S.-T. Khu and D. Butler \\ Centre for Water Systems, School of Engineering, Computing and Mathematics, University of Exeter, North \\ Park Road, Harrison Building, Exeter EX4 4QF, UK \\ (E-mail: g.fu@exeter.ac.uk;s.t.khu@exeter.ac.uk;d.butler@exeter.ac.uk)
}

\begin{abstract}
Simulation models are now available to represent the sewer network, wastewater treatment plant and receiving water as an integrated system. These models can be combined with optimisation methods to improve overall system performance through optimal control. Evolutionary algorithms (EAs) have been proven to be a powerful method in developing optimal control strategies; however, the intensive computational requirement of these methods imposes a limit on their application. This paper explores the potential of surrogate modelling in multiobjective optimisation of urban wastewater systems with a limited number of model simulations. A surrogate based method, ParEGO, is combined with an integrated urban wastewater model to solve real time control problems. This method is compared with the popular NSGA II, by using performance indicators: the hypervolume indicator, additive binary $\varepsilon$-indicator and attainment surface. Comparative results show that ParEGO is an efficient and effective method in deriving optimal control strategies for multiple objective control problems with a small number of simulations. It is suggested that ParEGO can greatly improve computational efficiency in the multiobjective optimisation process, particularly for complex urban wastewater systems.

Keywords Multiobjective optimisation; NSGA II; ParEGO; surrogate modelling; urban wastewater system
\end{abstract}

\section{INTRODUCTION}

There is growing recognition of the need to take an integrated approach to water management, which has led to the development of integrated models of the various hydraulic and water quality processes in the sewer system, treatment plant and receiving water body as a whole (Rauch et al., 2002; Butler and Schütze, 2005; Vanrolleghem et al., 2005). Integrated models provide us with the opportunity to control the urban wastewater system as a whole, and thus to achieve improved system performance through development of optimal control strategies; however, these models are normally very complex, and thus very challenging and expensive for optimisation methods.

Control of urban wastewater systems is usually regarded as a non-linear mathematical optimisation problem, and in many situations, a multiobjective optimisation problem ( $\mathrm{Fu}$ et al., 2008). Evolutionary algorithms (EAs) have been proven as promising to derive the optimal control strategies, compared with the conventional optimisation techniques (Rauch and Harremoës, 1999; Muschalla et al., 2006). However, EAs generally need tens of thousands of model simulations in order to reach the optimal control strategies. The computational burden makes EAs very inefficient and impractical for real time control problems in urban wastewater systems, which require a rapid decision making on selection of control strategies.

Surrogate modelling has been used for fitness approximation in evolutionary computation to improve computational efficiency, and a good summary was given by Jin (2005). The most used methods include polynomials, the kriging model, neural networks, and support vector machines. This paper explores the potential and benefit of a fast surrogate method, ParEGO (Knowles, 2006), for the multiobjective control problem in urban wastewater systems. This method is based on the 
popular kriging approach, the Design and Analysis of Computer Experiments (DACE), and can usually achieve a satisfying set of Pareto solutions within a few hundreds of objective evaluations. This method is compared with one of the state-of-the-art EAs, NSGA II (Deb et al., 2002), and is demonstrated by a case study.

\section{THE PAREGO ALGORITHM}

ParEGO is a surrogate based multiobjective optimisation method for expensive models (Knowles, 2006). It combines one of the kriging approaches, DACE, with genetic algorithms to achieve the best performance within a limited number of objective evaluations.

\section{DACE}

In the Kriging approach, the model $y$ with $n$ variables, $\mathbf{x}=\left(x_{1}, \cdots, x_{n}\right)$, is described as $y(\mathbf{x})=g(\mathbf{x})+z(\mathbf{x})$

where $g(\mathbf{x})$ is the regression term, usually a polynomial function, and $z(\mathbf{x})$ is the error term, represented by a Gaussian random function with zero mean and non-zero covariance. In the stochastic process, the errors for $N$ samples are related or 'correlated' and the correlation is related to the distance between the corresponding samples, and usually expressed as:

$$
R\left(\mathbf{x}^{(i)}, \mathbf{x}^{(j)}\right)=\exp \left[-\sum_{h=1}^{n} \theta_{h}\left|x_{h}^{(i)}-x_{h}^{(j)}\right|^{p_{h}}\right],\left(\theta_{h} \geq 0, p_{h} \in[1,2], \text { and } i, j=1, \quad \cdots, \quad N\right)
$$

where $\theta_{h}$ is a parameter measuring the importance of the variable $x_{h}, x_{h}^{(i)}$ and $x_{h}^{(j)}$ are the value of the variable $x_{h}$ in sample points $\mathbf{x}^{(i)}$ and $\mathbf{x}^{(j)}$, respectively. The covariance of $z(\mathbf{x})$ is denoted as $\operatorname{Cov}\left[z\left(\mathbf{x}^{(i)}\right), z\left(\mathbf{x}^{(j)}\right)\right]=\sigma^{2} \mathbf{R}\left[R\left(\mathbf{x}^{(i)}, \mathbf{x}^{(j)}\right)\right]$ where $z\left(\mathbf{x}^{(i)}\right)$ is Normal $\left(0, \sigma^{2}\right), i=1, \cdots, N$, and $\mathbf{R}$ is the symmetric correlation matrix for $N$ samples. It proves that modelling the correlation in this way is so powerful that the regression term $g(\mathbf{x})$ can be reduced to a simple constant term $\beta$, which is regarded as the mean of the stochastic process (Jones et al., 1998).

This model has a total of $2 n+2$ parameters: $\beta, \sigma^{2}, \theta_{1}, \cdots, \theta_{n}$ and $p_{1}, \cdots, p_{n}$. The maximum likelihood method can be used to estimate these parameters. The prediction for a point $\mathbf{x}^{*}$ can be calculated as

$$
\hat{y}=\hat{\beta}+\mathbf{r}^{\mathrm{T}}\left(\mathbf{x}^{*}\right) \mathbf{R}^{-1}(\mathbf{y}-\hat{\beta} \mathbf{I})
$$

where $\hat{\beta}$ is the estimated value of $\beta, \mathbf{y}$ is a vector of model outputs for the $N$ samples, $\mathbf{I}$ is a unit vector of length $N$, and $\mathbf{r}$ is the correlation vector between the error term at the predicted point and the error terms at the previously sampled points. The $i$ th element of $\mathbf{r}$ is $R\left(\mathbf{x}^{*}, \mathbf{x}^{(i)}\right)$.

One of the advantages of DACE is that a confidence interval of the prediction can be obtained, which is explicitly used by ParEGO to guide the search. A MATLAB toolbox developed by Lophaven et al. (2002) is used for implementing the DACE model.

\section{Implementation of ParEGO}

The implementing process of ParEGO is shown in Figure 1. An internal genetic algorithm is used to search for the solution that maximizes the expected improvement, and to update the solution set, which consists of solutions evaluated by the real objective functions. For a detailed description of ParEGO, the reader is referred to Knowles (2006). 


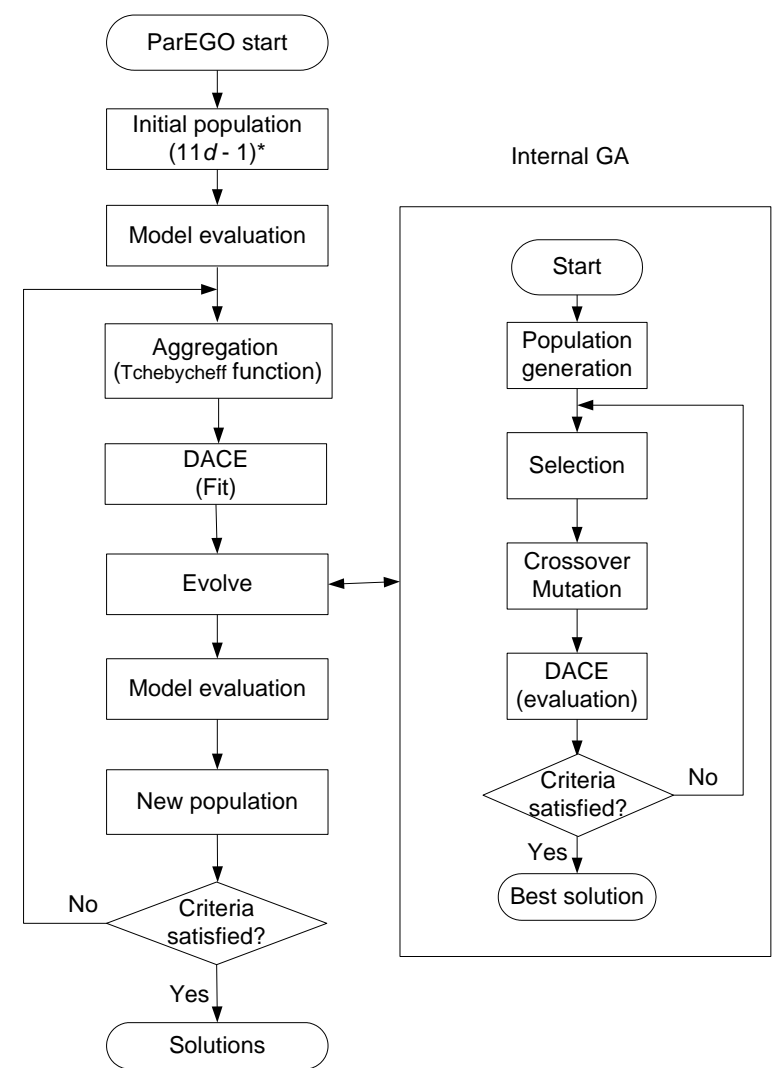

Figure 1 Flow chart of ParEGO. $* 11 d-1$ is the size of the initial population, and $d$ the number of decision variables.

ParEGO is basically an aggregation-based algorithm, and the non-linear Tchebycheff function was used to combine the $m$ objectives into one single objective

$f=\max _{j=1}^{m} \lambda_{j} f_{j}+\rho \sum_{j=1}^{m} \lambda_{j} f_{j}$

Where $f_{j}$ and $\lambda_{j}(j=1,2, \cdots, m)$ are the $j$ th normalized objective value and its weight, respectively, and $\rho$ is a small positive parameter and was set to 0.05 according to Knowles (2006). The objectives are assumed to be simultaneously minimized in the aggregation function, and thus maximization objectives should be converted to minimization. In order to explore the whole region of the Pareto front, a varying weight vector is used in ParEGO, and is drawn randomly from the evenly distributed vector set,

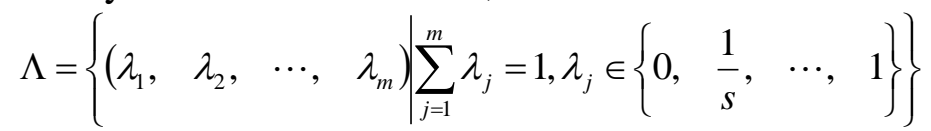

The total number of vectors in the above set is determined by $|\Lambda|=\left(\begin{array}{c}s+m-1 \\ m-1\end{array}\right), s$ is set to 10 for the two-objective case in this paper.

\section{PERFORMANCE INDICATORS}

Performance indicators are used to assess and compare the properties of an approximation set derived from multiobjective evolutionary algorithms. Two quantitative indicators, i.e., hypervolume indicator and additive binary $\varepsilon$-indicator, were selected to compare the performance of ParEGO with NSGA II. Additionally, attainment surface plots were also used for visual performance comparison. 


\section{Hypervolume indicator}

This indicator, also known as the $S$ metric or the Lebesgue measure, measures the size of the region of objective space dominated by a set of solutions. The hypervolume not only indicates the closeness of the solutions to the optimal set, but also captures the spread of the solutions over the objective space.

Several algorithms exist for calculating hypervolume, such as, the inclusion-exclusion (Wu and Azarm, 2001), LebMeasure (Fleischer, 2003), and HSO (While et al., 2006). The HSO algorithm is used due to its computational efficiency. In calculating the region, a reference solution must be chosen, which should be dominated by every solution in the Pareto set. In this research, the reference solution is chosen from all the Pareto solutions obtained from each run.

\section{Additive binary $\varepsilon$-indicator}

This indicator was first defined by Zitzler et al. (2003) as follows: for a minimization problem with $m$ positive objectives, a solution $z^{1}=\left(z_{1}^{1}, z_{2}^{1}, \cdots, z_{m}^{1}\right)$ is said to $\varepsilon$-dominate another solution $z^{2}=\left(z_{1}^{2}, \quad z_{2}^{2}, \cdots, \quad z_{m}^{2}\right)$, denoted as $z_{i}^{1} \leq_{\varepsilon+} z_{i}^{2}$, if and only if $\forall 1 \leq i \leq m: z_{i}^{1} \leq \varepsilon+z_{i}^{2}$

Then a pair of numbers $\left(I_{A}, I_{B}\right)$ is defined as the binary $\varepsilon$-indicator

$$
\begin{aligned}
& I_{A}=I_{\varepsilon+}(A, B)=\inf _{\varepsilon \in \Re}\left\{\forall z^{2} \in B \quad \exists z^{1} \in A: z^{1} \leq_{\varepsilon+} z^{2}\right\} \\
& I_{B}=I_{\varepsilon+}(B, A)=\inf _{\varepsilon \in \mathcal{R}}\left\{\forall z^{2} \in A \exists z^{1} \in B: z^{1} \leq_{\varepsilon+} z^{2}\right\}
\end{aligned}
$$

for a pair of Pareto set $A$ and $B$. The Pareto set $A$ is strictly better than $B$ if $I_{A} \leq 0$ and $I_{B}>0$, and the two sets are incomparable if $I_{A}>0$ and $I_{B}>0$. However, $A$ could be interpreted to be better than $B$ in a weaker sense if $I_{A}<I_{B}$ (Knowles, 2006).

In general, higher hypervolumes and smaller binary $\varepsilon$-indicators indicate a better performance. However, the two algorithms considered are stochastic search methods and involve some randomness, for example, from the genetic operators and sampling of the initialisation stage. In order to filter the impact of randomness, 10 optimisation runs were conducted for reliable comparisons of the two algorithms.

\section{RESULTS AND DISCUSSION}

A case study is used to demonstrate the potential of ParEGO for multiobjective control of urban wastewater systems with a limited number of model simulations. Two experiments with 160 (150 for ParEGO) and 260 objective evaluations have been conducted for comparison with NSGA II. The parameters for these two algorithms are set according to Knowles (2006).

\section{Case study}

The approach is demonstrated by an integrated case study, consisting of a combined sewer system, a treatment plant and receiving river, as shown in Figure 2. The catchment was first defined by Schütze (1998) and has been studied in detail for real time control optimisation (Schütze et al., 2002; Butler and Schütze, 2005; Fu et al., 2008).

The sewer system has seven sub-catchments with a total area of $725.8 \mathrm{ha}$, and four on-line passthrough storage tanks linked to sub-catchments 2, 4, 6 and 7, respectively. The wastewater treatment plant includes an off-line pass-through storm tank, a primary clarifier, aerator, and secondary clarifier. The treatment plant effluent and storm tank overflow are discharged to the river 
at Reach 10, and CSO discharges at Reach 7. This urban system was simulated using the SIMBA tool in the MATLAB/SIMULINK environment (IFAK, 2005).

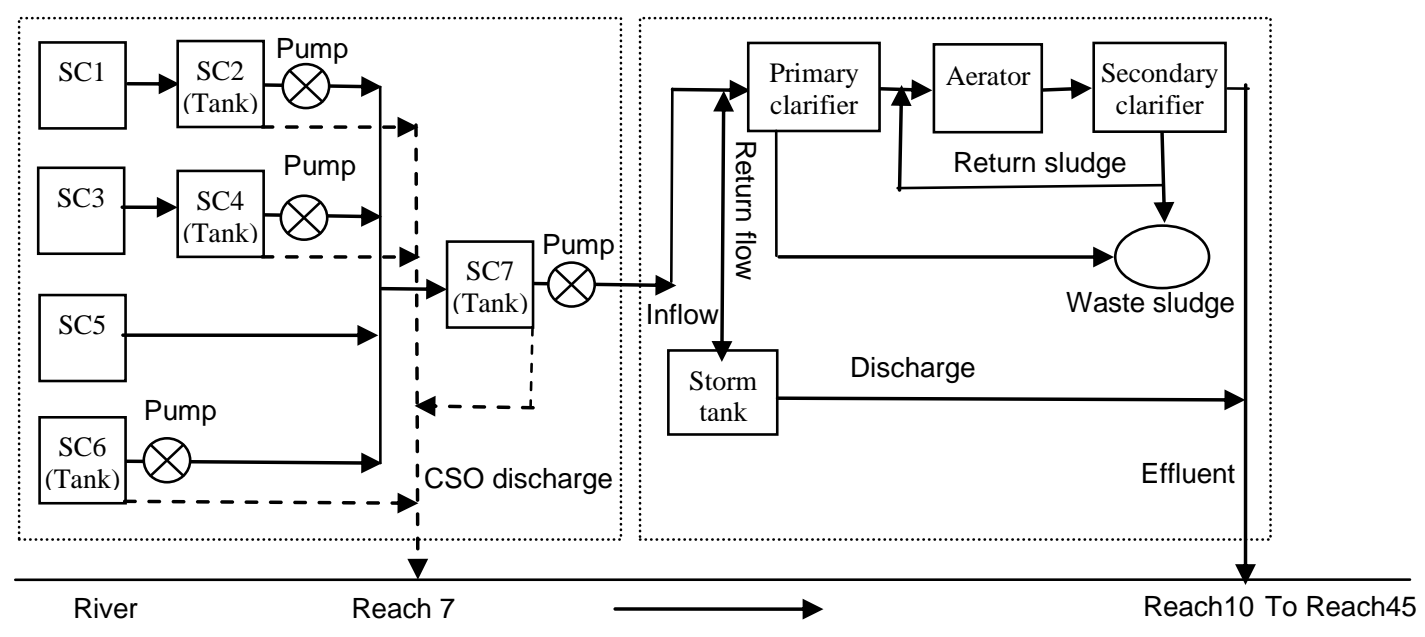

Figure 2 Schematic representation of the integrated urban wastewater system. SCx represents the xth sub-catchment, and the dash lines show CSO discharges from the storage tanks.

The optimisation problem is defined to improve the receiving water quality through optimal system control. Based on sensitivity analysis, the chosen control variables include the maximum outflow rate of the storage tank linked to sub-catchment 7, the maximum inflow rate to the treatment plant, the threshold starting to empty the storm tank and its emptying flow rate, and the return sludge rate. The receiving water quality is represented by two water quality indicators, i.e., minimum DO concentration (DO-M) and maximum ammonium concentration (AMM-M) along all the river reaches during the entire simulation period. Thus, the objectives are minimisation of AMM-M and maximisation of DO-M.

\section{Hypervolume and additive binary $\varepsilon$-indicators}

ParEGO and NSGA II were implemented 10 times for comparison, so the hypervolume and binary $\varepsilon$-indicators were calculated for the Pareto set from each run, and the standard deviations are also computed for comparison.
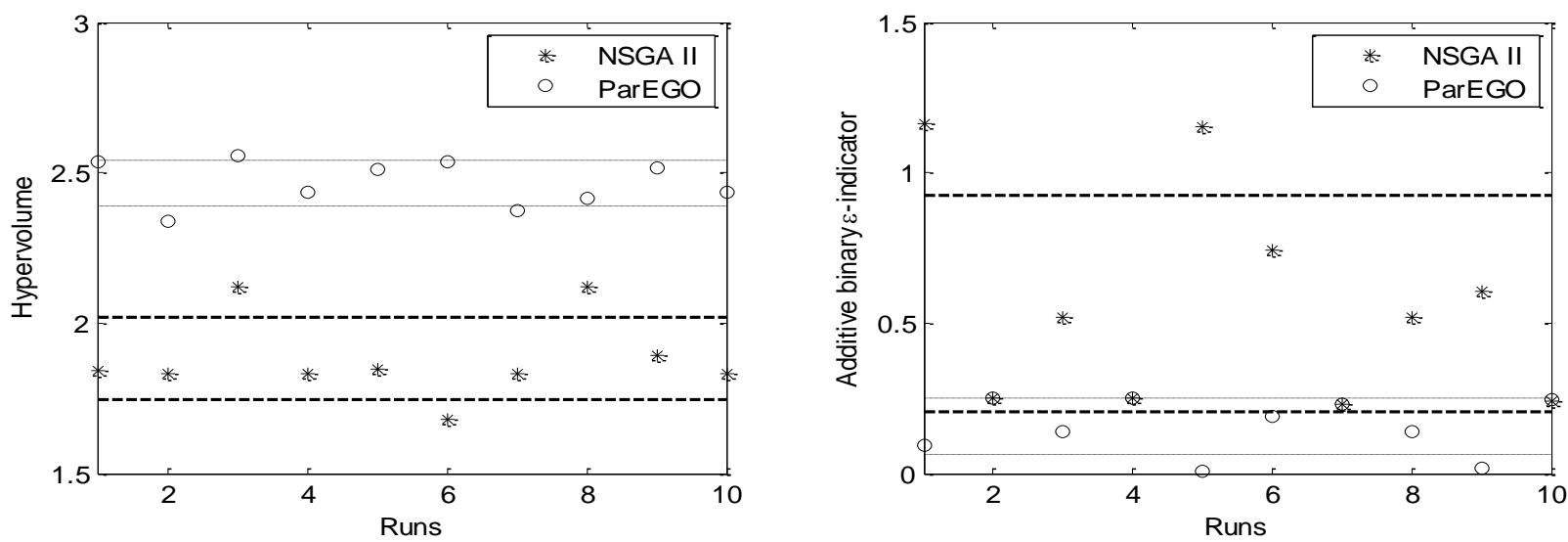

Figure 3 Hypervolume and additive binary $\varepsilon$-indicators for 160 (NSGA II) and 150 (ParEGO) objective evaluations. The dashed lines indicate the standard deviation for NSGA II results, and the dotted lines for ParEGO results. 
Figures 3 and 4 show the hypervolume and additive binary $\varepsilon$-indicators for 160 (150 for ParEGO) and 260 objective evaluations, respectively. For the hypervolume indicator, ParEGO can achieve a higher value for each run, which means a larger region in the objective space. For the additive binary $\varepsilon$-indicator, ParEGO weakly dominates NSGA II for all the runs with 260 evaluations. However, a relatively equivalent performance is achieved for the runs with 160/150 evaluations, considering that NSGA II achieves a smaller value in four cases. For both of the indicators, the standard deviations for ParEGO are always smaller than those of NSGA II, so it shows that ParEGO is more reliable.
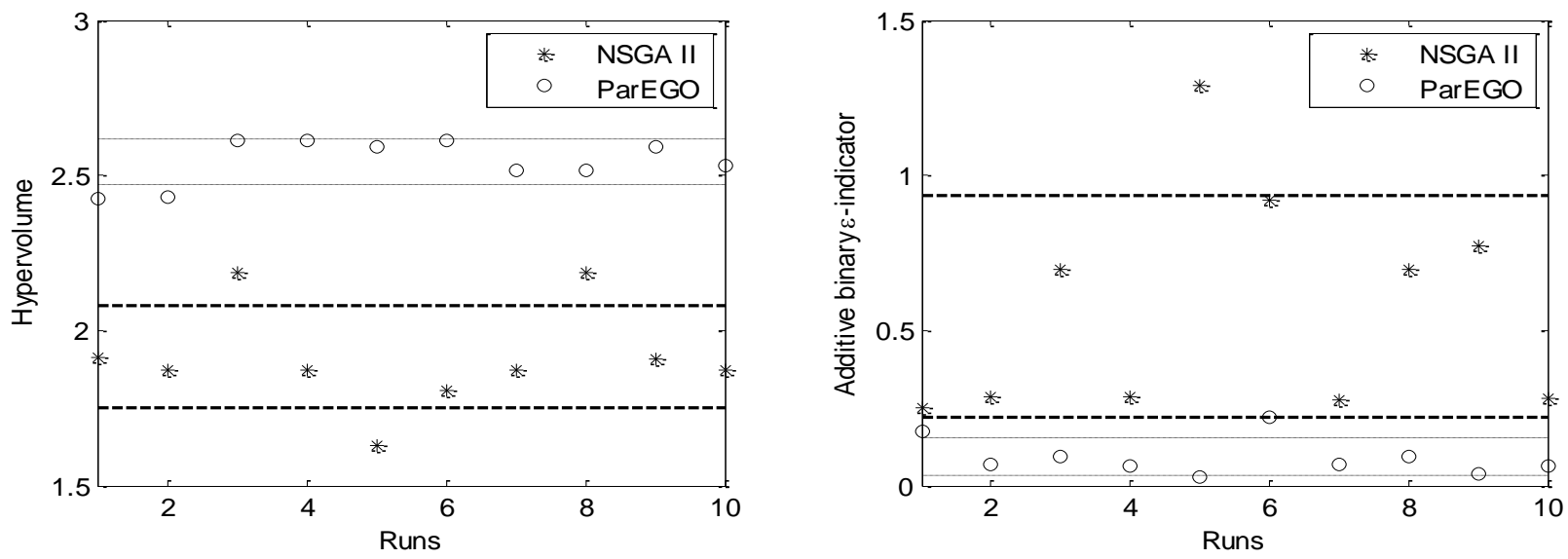

Figure 4 Hypervolume and additive binary $\varepsilon$-indicators for 260 objective evaluations. The dashed lines indicate the standard deviation for NSGA II results, and the dotted lines for ParEGO results.

\section{The attainment surfaces}

An attainment surface can show the division of the objective space by a set of the Pareto solutions. The best attainment surface from multiple runs visualises the biggest objective space that is achieved in all the runs, so it gives a good indication about the performance of an algorithm. The best surfaces for NSGA II and ParEGO were obtained from the 10-run results, as shown in Figure 5. The plots show that ParEGO can dominate a larger space, particularly in the left hand side of the space. This probably was affected by the weights in the aggregation function, which gives a greater exploration on the space of the AMM-M objective.
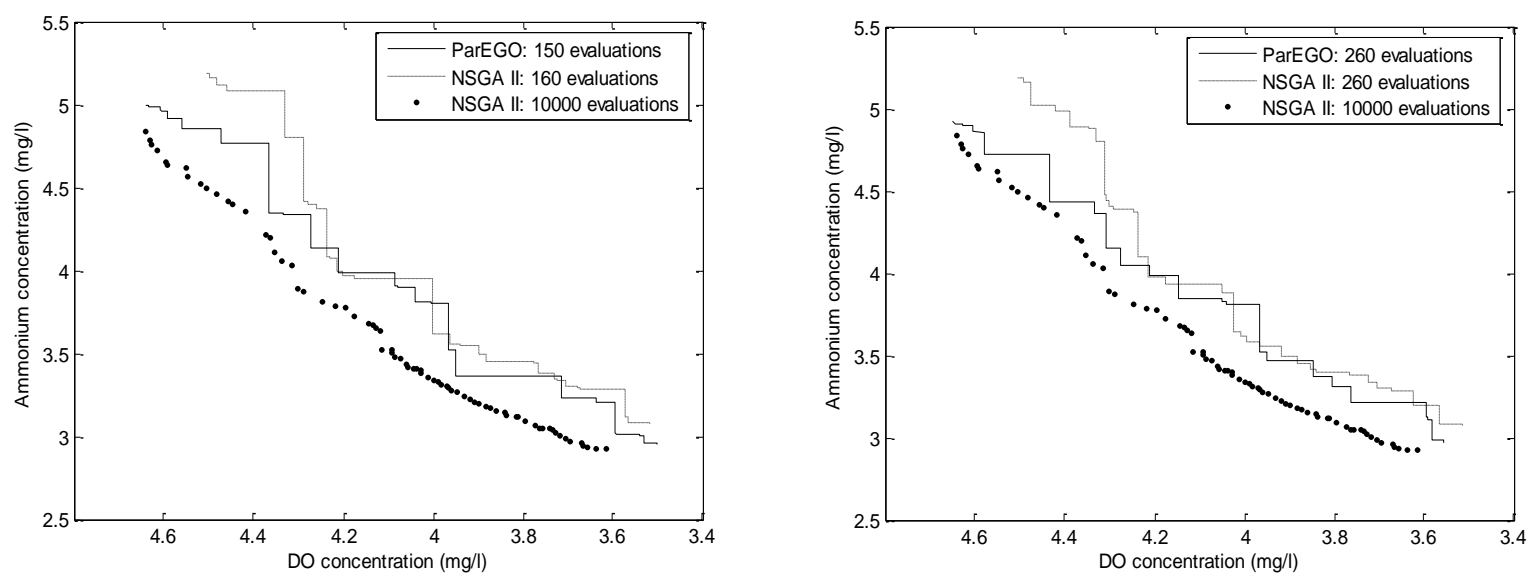

Figure 5 The best attainment surfaces for ParEGO and NSGA II.

A visual insight into the performance of ParEGO can also be shown by comparing the best surfaces with the Pareto front from a full NSGA II run with 10,000 objective evaluations. It can be seen from Figure 5 that the best surfaces from ParEGO give an overall good approximation to the Pareto front. 
In some regions of the objective space, the algorithm performs better with a very close approach to the Pareto front, while some other parts have a poorer approximation. This might be due to the use of uniformly random scalarizing weight vectors to aggregate the two objectives, which could not automatically result in the best distribution of non-dominated solutions when some parts of the Pareto front are harder to find than others (Knowles, 2006).

\section{Computation time}

For the case of 260 model simulations, the average time of 10 runs is 96 minutes for NSGA II, and 102 minutes for ParEGO on a P4 CPU $2.40 \mathrm{GHz}$ PC. ParEGO takes slightly more time due to the DACE model building and local search process. However, this additional time is negligible compared to the total computation time of the integrated model, and this will be more obvious for a long-term simulation or more expensive model.

\section{CONCLUSIONS}

This paper explores the potential and benefit of a fast surrogate method, ParEGO, for multiobjective optimisation of urban wastewater systems with a limited number of model simulations. This method is demonstrated by an integrated real time control problem in which the receiving water quality indicator (i.e., the minimum DO and maximum ammonium concentrations) are directly used as control objectives. Compared with NSGA II, ParEGO can achieve a better performance, particularly for the runs with 260 objective evaluations, and it has less variation in performance. Further, ParEGO also gives a good approximation to the set of Pareto solutions derived by NSGA II with 10,000 objective evaluations. Results illustrate that for the optimal control problem in the urban wastewater system ParEGO is an efficient and effective method in deriving optimal control strategies.

\section{ACKNOWLEDGEMENT}

The study is partly funded by UK EPSRC grant no GR/S86846/01 and is also part of the Integrative Systems and the Boundary Problem (ISBP) project (www.tigress.ac/isbp), supported by the European Union's Framework 6 Programme.

\section{REFERENCES}

Butler D. and Schütze M. (2005). Integrating simulation models with a view to optimal control of urban wastewater systems. Environmental Modelling and Software, 20, 415-426.

Deb K., Pratap A, Agarwal S. and Meyarivan T. (2002). A fast and elitist multi-objective genetic algorithm: NSGA-II. IEEE Transaction on Evolutionary Computation, 6(2), 181-197.

Fleischer M. (2003). The measure of Pareto optima: Applications to multi-objective metaheuristics. In: Evolutionary Multiobjective Optimisation. ser. Lecture Notes in Computer Science, C. M. Fonseca et al. (ed.), Springer-Verlag, Berlin, vol. 2632, pp. 519-533.

Fu G., Butler D. and Khu S.T. (2008). Multiple objective optimal control of integrated urban wastewater systems. Environmental Modelling and Software, 23(2), 225-234.

IFAK, (2005). SIMBA 5.0: simulation of wastewater systems, user's guide. Institut für Automation und Kommunikation e.V. Magdeburg, Germany.

Jin Y. (2005). A comprehensive survey of fitness approximation in evolutionary computation. Soft Computing, 9, 3-12.

Jones D., Schonlau M. and Welch W. (1998). Efficient global optimisation of expensive black-box functions. Journal of Global Optimisation, 13, 455-492.

Knowles J., (2006). ParEGO: A hybrid algorithm with on-line landscape approximation for expensive multiobjective optimisation problems. IEEE Transactions on Evolutionary Computation, 10(1), 50-66.

Lophaven S.N., Nielsen H.B. and Søndergaard J. (2002). DACE - A Matlab Kriging Toolbox, Version 2.0. Technical Report IMM-TR-2002-12, Technical University of Denmark. http://www2.imm.dtu.dk/ hbn/dace (accessed 24 October 2008). 
Muschalla D., Schrpter K., and Schütze M. (2006). Multi-objective evolutionary algorithms in the field of urban drainage. Seventh International Conference on Hydroinformatics, Nice, France.

Rauch W., and Harremoës P., (1999). On the potential of genetic algorithms in urban drainage modelling. Urban Water, 1, 79-89.

Rauch W., Bertrand-Krajewski J.L., Krebs P., Mark O., Schilling W., Schütze M., and Vanrolleghem P.A. (2002). Mathematical modelling of integrated urban drainage systems. Water Science and Technology, 45(3), 81-94.

Schütze M. (1998). Integrated Simulation and Optimum Control of the Urban Wastewater System. PhD thesis. Department of Civil Engineering; Imperial College of Science, Technology and Medicine, University of London, London.

Schütze M., Butler D. and Beck M.B. (2002). Modelling Simulation and Control of Urban Wastewater Systems. Springer, Berlin.

Vanrolleghem P.A., Benedetti L., and Meirlaen J. (2005). Modelling and real-time control of the integrated urban wastewater system. Environmental Modelling \& Software, 20(4), 427-442.

While L., Hingston P., Barone L., and Huband S. (2006). A faster algorithm for calculating hypervolume. IEEE Transactions on Evolutionary computation, 10(1), 29-38.

Wu J. and Azarm S. (2001). Metrics for quality assessment of a multiobjective design optimisation solution set. $J$. Mechanical Design, 123, 18-25.

Zitzler E., Thiele L., Laumanns M., Fonseca C.M., and Fonseca V.G. (2003). Performance assessment of multiobjective optimizers: An analysis and review. IEEE Transactions on Evolutionary Computation, 7(2), 117-132. 